\title{
Antibiotic Prescribing Pattern in Neonates of Seventeen Iranian Hospitals
}

\author{
Alireza Fahimzad, ${ }^{1}$ Zahra Eydian,,${ }^{2}{ }^{*}$ Abdollah Karimi, ${ }^{1}$ Farideh Shiva, ${ }^{1}$ Shahnaz Armin, ${ }^{1}$ Roxana \\ Mansour Ghanaei, ${ }^{1}$ Fatemeh Fallah, ${ }^{1}$ Sedigheh Rafiei Tabatabaei, ${ }^{1}$ Fariba Shirvani,, ${ }^{1}$ Mohammad \\ Rahbar, ${ }^{3,4}$ Shirin Sayyahfar, ${ }^{3}$ Manijeh Kahbazi, ${ }^{4}$ Aliakbar Rahbarimanesh, ${ }^{5}$ Reza Arjmand, ${ }^{6}$ Anahita \\ Sanaei Dashti, ${ }^{7}$ Babak Abdinia, ${ }^{8}$ Masomeh Abedini, ${ }^{9}$ Hossein Hidari, ${ }^{10}$ Keyghobad Ghadiri, ${ }^{11}$ \\ Aminsadat Sharif,, ${ }^{12}$ Elham Shafighi Shahri, ${ }^{13}$ Zeynab Shariat, ${ }^{14}$ Ali Nejadian, ${ }^{15}$ Mona Moshayedi, ${ }^{16}$ \\ Samaneh Fatholahpoor, ${ }^{17}$ Hamid Alizadeh ${ }^{18}$ and Reza Entezari Heravi ${ }^{19}$ \\ ${ }^{1}$ Pediatric Infections Research Center, Research Institute For Children Health, Shahid Beheshti University of Medical Sciences, Tehran, Iran \\ ${ }^{2}$ Department of Pediatrics, Zabol University of Medical Sciences, Zabol, Iran \\ ${ }^{3}$ Department of Microbiology, Iranian Reference Health Laboratory, Ministry of Health and Medical Education, Tehran, Iran \\ ${ }^{4}$ Antimicrobial Resistance Research Center, Iran University of Medical Sciences, Tehran, Iran \\ ${ }^{5}$ Department of Pediatrics, Aliasghar Children Hospital, Iran University of Medical Sciences, Tehran, Iran \\ ${ }^{6}$ Tuberculosis and Pediatrics Infectious Disease Research Center, Arak University of Medical Sciences, Arak, Iran \\ ${ }^{7}$ Department of Pediatric Infectious Diseases, Bahrami Children Hospital, Tehran University of Medical Sciences, Tehran, Iran \\ ${ }^{8}$ Department of Pediatrics, Alborz University of Medical Sciences, Karaj, Iran \\ ${ }^{9}$ Professor Alborzi Clinical Microbiology Research Center, Shiraz University of Medical Sciences, Shiraz, Iran \\ ${ }^{10}$ Department of Pediatrics, Faculty of Medicine, Tabriz University of Medical Sciences, Tabriz, Iran \\ ${ }^{11}$ Department of Pediatrics Faculty of Medicine, Sanandaj University of Medical Sciences, Sanandaj, Iran \\ ${ }^{12}$ Pediatric Medicine Research Center, Qom University of Medical Sciences, Qom, Iran \\ ${ }^{13}$ Nosocomial Infection Research Center, Kermanshah University of Medical Sciences, Kermanshah, Iran \\ ${ }^{14}$ Pediatric Department, Faculty of Medicine, Hamadan University of Medical Sciences, Hamadan, Iran \\ ${ }^{15}$ Children and Adolescent Health Research Center, Zahedan University of Medical Sciences, Zahedan, Iran \\ ${ }^{16}$ Department of Pediatrics Infectious Disease and Tropical Medicine Research Center, Isfahan University of Medical Sciences, Isfahan, Iran \\ ${ }^{17}$ Department of Pediatric Infectious Disease, Abuzar Children Medical Center Hospital, Ahvaz Jundishapour University of Medical Sciences, Ahvaz, Iran \\ ${ }^{18}$ Antimicrobial Resistant Nosocomial Infection Research Center, Mazandaran University of Medical Sciences, Sari, Iran \\ ${ }^{19}$ Non-Communicable Pediatric Diseases Research Center, Babol University of Medical Sciences, Babol, Iran \\ ${ }^{20}$ Department of Pediatrics, Gilan University of Medical Sciences, Rasht, Iran \\ ${ }^{21}$ Faculty of Pharmacy, Zabol University of Medical Sciences, Zabol, Iran \\ "Corresponding author: Zahra Eydian, Department of pediatrics, Zabol University of Medical Sciences, Zabol, Iran. Tel: +98-54322951215, E-mail: zahra.eydian@yahoo.com
}

Received 2016 November 27; Revised 2017 February 12; Accepted 2017 February 28.

\begin{abstract}
Background: The association between the use of antibiotics and bacterial resistance has obviously been established and it seems to be a significant problem for public health. It is clear that irrational use and high rates of antibiotic prescription are associated with increased bacterial resistance. As antibiotics are the class of drugs commonly prescribed in neonatal and Neonatal Intensive Care Unit (NICU) wards, neonates are at high risk of opportunistic or nosocomial infections due to prolonged hospitalization and immunosuppressed condition.

objectives: It is essential for antibiotic prescription patterns to be evaluated periodically for rational use. Therefore, the present study was carried out to identify the prescribing patterns of antibiotics in neonatal and NICU wards of 17 different Iranian hospitals.

Methods: The study was done during 1 calendar week between January and February 2014. All in-patients admitted to the NICU and neonatal units of 17 Hospitals in 15 Iranian cities were included. Relevant data of all neonates receiving an antibiotic at 8 am on the day of the study was collected by trained members of the study team; age of the patients, type and number of administered antibiotics, route of administration, underlying disease, and indication for use were documented.

Results: The total number of neonatal in-patients was 366 on the day of the study. Mean age of infants was $9.2 \pm 3.9$ days, with $54.4 \%$ being male. Overall, 264 patients (72.1\%) were being given one or more antimicrobials; the proportion of patients receiving antibiotics ranged from $21.4 \%$ to $100 \%$ in different neonatal units. Most frequent antibiotics used were ampicillin (25.6\%), vancomycin (16.4\%), amikacin (10.6\%), and cefotaxime (9.74\%). Parenteral route was used in 255 cases, (96.6\%); 110 (41.7\%) antibiotic courses were prescribed for community-acquired infections, 29 (11\%) for hospital-acquired infections, 42 (15.9\%) for pre- or post-operative prophylaxis, and 83 ( $31.4 \%$ ) for medical prophylaxis. Empirical antibiotics were administered to 255 newborns (96.6\%); $96.2 \%$ of patients received combination therapy.

Conclusions: High use of empirical and prophylactic antibiotics, overuse of vancomycin and third generation cephalosporins are of concern and contrary to recommended therapies. Therefore, guidelines for the use of antibiotics in neonates are required and a larger number of studies are needed.
\end{abstract}

Keywords: Neonates, Empirical Therapy, Antibiotics, Prophylaxis, Combination Therapy

\section{Background}

Antimicrobial resistance (AMR) is a serious worldwide problem that has been named by the world health organi-

Copyright (c) 2017, Archives of Pediatric Infectious Diseases. This is an open-access article distributed under the terms of the Creative Commons Attribution-NonCommercial 4.0 International License (http://creativecommons.org/licenses/by-nc/4.0/) which permits copy and redistribute the material just in noncommercial usages, provided the original work is properly cited. 
zation (WHO), as a global crisis (1). Indiscriminate antibiotic consumption is recognized as the prime cause of escalating bacterial resistance $(2,3)$.

Antibiotics are among the most common medications prescribed for hospitalized patients, especially in children and neonates, and it has been estimated that about $1 \%$ of newborns in industrialized countries are administered antibiotics (4). Although a global increase in the number of resistant bacteria necessitates the use of potent antibiotics to combat serious infections and the use of antibiotics is necessary and lifesaving in ill infants and neonates, irrational antimicrobial prescription in situations where antibiotics are misused, is widespread globally, especially in developing countries (5-7).

In order to promote rational antimicrobial prescription and to prevent the emergence of resistant microorganisms, it is crucial to determine the pattern of antibiotic prescription for inpatients and publish guidelines for proper usage. Point-prevalence surveys (PPS) have been used to estimate the total prevalence of healthcareassociated infections and also to provide information about antimicrobial use for acute care in hospitals (8, 9). Since 2006, the European surveillance of antimicrobial consumption (ESAC) has performed a number of PPS on the use of antibiotics in hospitals from different European and non-European countries (10-12). These studies were performed on adults. On the successful completion of these projects, a similar plan was developed for children, surveying antimicrobial use in children and neonates, with the name of "antibiotic resistance and prescribing in European children" or "ARPEC" $(13,14)$.

The aim of the present study was to perform a point prevalence survey on the rate and pattern of antibiotic prescription in neonatal units of 17 hospitals from 15 cities of Iran, to identify targets for quality improvements in antimicrobial prescription patterns.

\section{Methods}

The study was conducted during one calendar week between January and February 2014, in 17 Iranian hospitals. The analyses were restricted to hospitals with neonatal and NICU wards. Medical records of neonatal in-patients, aged less than one month, were reviewed by members of the study team, comprised of pediatricians or pediatric infectious disease specialists. All neonates recruited in this study were admitted to the hospital at least 24 hours before the survey and were in-patients at 8 am on the day of the survey. The following information was collected from infants receiving antimicrobials on the day of the survey, age, antibiotic agent and its indication, underlying disease, empirical or targeted base on cultures, route of administra- tion, therapeutic or prophylactic use of antibiotics. Usage of more than one antibiotic for patients was considered as combination therapy. All outpatient neonates and children with an age of over one month were excluded from this study. Administration of prophylactic antimicrobials in the previous 24 hours for surgical patients was recorded as a single dose, multi-dose in one day, and administration of antibiotic for more than one day.

\section{Results}

A total of 366 newborns from 17 hospitals were included in the study from 15 Iranian cities. All hospitals were public and equipped with neonatal and NICU wards. Among 17 hospitals, there were 6 pediatric hospitals, two of which were equipped with pediatric surgical wards. Eleven out of 17 hospitals were general hospitals with medical, surgical, and maternity wards. Mean age of infants was $9.2 \pm 3.9$ days, with $54.4 \%$ being male. Two-hundred and sixty-four patients (72.1\%) were given one or more antimicrobials. The proportion of patients receiving antibiotics ranged from $21.4 \%$ to $100 \%$ at different neonatal units.

The number of admitted patients, in all neonatal and NICU wards of 17 Iranian hospitals, is shown on Figure 1. The most frequent antibiotics used were ampicillin (25.6\%), vancomycin (16.4\%), amikacin (10.6\%), and cefotaxime (9.74\%) (Figure 2). Parenteral route was used for administration of the antibiotics in 255 cases (96.6\%). Of the 264 antimicrobial prescriptions, 110 (41.7\%) were prescribed for community-acquired infections, 29 (11\%) for hospital-acquired infections, 42 (15.9\%) for pre- or postoperative prophylaxis, and 83 (31.4\%) for medical prophylaxis on admission to the neonatal unit. Two-hundred and fifty-five newborns (96.6\%) were administered empirical antibiotics and 9 (3.4\%) received targeted antibiotic therapy based on cultures; $96.2 \%$ of the patients received combination therapy and 3.8\% were prescribed a single antibiotic (Table 1). Out of 264 neonates that received antibiotics, 54 patients $(20.4 \%)$ had no underlying diseases while the rest either had a history of respiratory problems (33.3\%), maternal risk factors, such as premature rupture of membranes (7.9\%), or other congenital or acquired underlying co-morbidities (Figure 3).

\section{Discussion}

The major aim of this study was to evaluate prescription patterns of antibiotics to avoid irrational use of antimicrobials as they have been identified as a risk factor for resistant pathogens (12).

This point prevalence survey (PPS) of 17 hospitals in 15 cities revealed a wide variation of antibiotic use, of $21.4 \%$ 


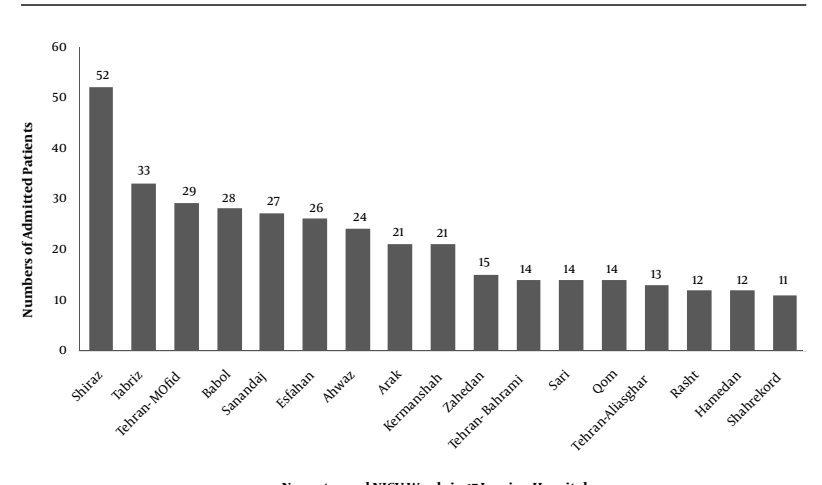

Figure 1. Total Admitted Patients in Neonatal and Neonatal Intensive Care Unit Wards of 17 Iranian Hospitals

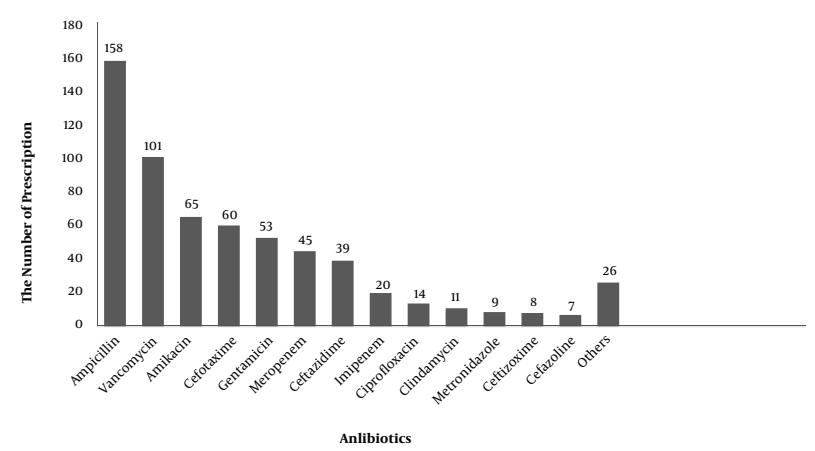

Figure 2. Total Number of Antimicrobial Prescription in Neonatal and Neonatal Intensive Care Unit Wards of 17 Iranian Hospitals

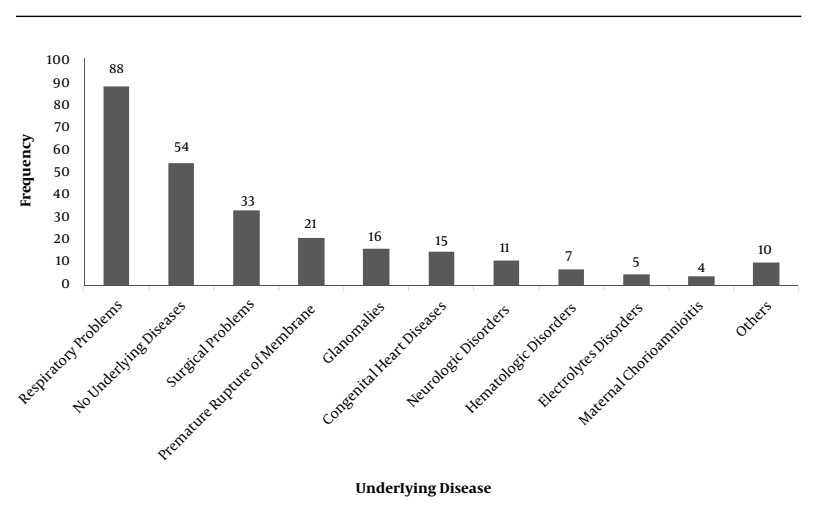

Figure 3. Frequency of Underlying Diseases Among All Neonates Admitted to Neonatal and Neonatal Intensive Care Unit Wards of 17 Iranian Hospitals

to $100 \%$ at different neonatal units. Discrepancy in the rate of antibiotic usage could be explained by the fact that higher figures are reported from neonatal intensive care units (NICUs) as almost 100\%, thereby admitting very sick infants, many of whom need respiratory support. Point
Table 1. Distribution of Neonates by Antimicrobial Prescription Characteristics in 17 Iranian Hospitals

\begin{tabular}{|c|c|}
\hline Description & Frequency (\%) \\
\hline \multicolumn{2}{|l|}{ Gender } \\
\hline Male & $199(54.5 \%)$ \\
\hline Female & $167(45.6 \%)$ \\
\hline Acquired infection from the society & $110(41.7 \%)$ \\
\hline Acquired infection from the hospital & $29(11.0 \%)$ \\
\hline Surgical prophylaxis & $42(15.9 \%)$ \\
\hline Medical prophylaxis & $83(31.4 \%)$ \\
\hline \multicolumn{2}{|l|}{ Route of administration } \\
\hline Parenteral & $255(96.6 \%)$ \\
\hline Oral & $9(3.4 \%)$ \\
\hline \multicolumn{2}{|l|}{ Type of Treatment } \\
\hline Combination therapy & $254(96.2 \%)$ \\
\hline Monotherapy & $10(3.8 \%)$ \\
\hline \multicolumn{2}{|l|}{ Antibiotic prescription } \\
\hline Empiric & $255(96.6 \%)$ \\
\hline Targeted & $9(3.4 \%)$ \\
\hline Pre-term neonates & $117(44.3 \%)$ \\
\hline \multicolumn{2}{|l|}{ Ventilated neonates } \\
\hline Invasive & $44(16.7 \%)$ \\
\hline Non-invasive & $4(1.5 \%)$ \\
\hline
\end{tabular}

prevalence surveys done for pediatric and neonatal patients from other areas have also reported a wide variance between different regions and also at different wards of the same hospital $(11,15)$. A widespread PPS performed in 50 European and 23 non-European hospitals of 23 countries (14 European and 9 non-European countries) revealed a very high use of broad spectrum antibiotics in pediatric and neonatal in-patients from non-European countries as compared to Europe (11). A PPS performed on 6 newborn units in Australia reported that $46 \%$ of inpatients were administered at least 1 antibiotic, (16). In another study performed at a level III NICU, on 1607 infants during a period of 14 months, $72 \%$ of neonates were given 1 or more courses of antibiotics; these figures are comparable to the current study (17).

Sepsis was a major cause of death in newborns, during year 2013; out of 2.8 million reported neonatal deaths, 0.42 million were attributed to neonatal sepsis (18). Since signs of sepsis are non-specific in neonates, empirical antibiotics are usually started in newborns admitted with suspected sepsis; duration of therapy depends not only on culture results, but also on clinical assessment of the pa- 
tient. Even if the culture results are negative, physicians may continue administering empiric treatment if the general condition of the patient and/or other laboratory investigations suggests the possibility of an ongoing infectious process. However, administering empiric antibiotics is not innocuous and may be associated with serious adverse effects that include alteration of gut micro biota, colonization with antibiotic resistant bacteria, late-onset sepsis, necrotizing enterocolitis, invasive candidiasis, and increased risk of mortality (17-19). In the current study, almost all antimicrobial prescriptions ( $>96 \%$ ) were empirical on the day of the survey while less than $4 \%$ were targeted therapy for specific proven infections; these figures are similar to another study, in which only $5 \%$ of antimicrobials were given for culture-proven infections (17). Since the current study was a point prevalence study, the authors do not know if empiric antibiotics initiated for suspected sepsis were continued for prolonged periods or stopped within 48 to 72 hours, if the culture results were negative; other studies have reported varying figures of continued antibiotic use, despite negative culture results, especially in preterm infants $(17,19)$. Most frequently used antibiotics in the surveyed neonatal units were ampicillin, vancomycin, amikacin and cefotaxime, gentamicin, and meropenem and ceftazidime, in the mentioned order. Antibiotic prescriptions in different countries or even in different NICUs in the same country may be widely variable as to the type of prescribed antibiotics, depending on the prevalent bacterial flora in the unit, antibiotic resistance patterns, age of the neonate (EOS vs. LOS), origin of infection, (community-acquired vs. hospital acquired), and physician preferences. The World Health Organization recommends intravenous ampicillin together with gentamicin, as an initial empirical treatment for neonatal sepsis, while third generation cephalosporins are used if the patient does not respond within 48 hours (18). Penicillin, gentamicin, and ampicillin were the most frequently used antibiotics for neonates in many studies; vancomycin, however, was not used often and was administered only in $1 \%$ of patients in one of the studies. Moreover, meropenem and cefotaxime were among the least-used antibiotics (16, 17,20 ). There is a paucity of randomized controlled trials to approve the most optimal antibiotic regimens for the treatment of neonatal sepsis, yet most experts recommend the empirical use of ampicillin together with an aminoglycoside for EOS and anti-staphylococcal penicillin together with an aminoglycoside for LOS. Vancomycin should be reserved for treatment of Methicillin Resistant Staphylococci (MRSA), in unstable newborns from areas where MRSA is prevalent, or in the case of proven sepsis with coagulasenegative staphylococci (CoNS) $(4,21)$. These recommendations hold true for most industrialized countries, how- ever, most deaths from neonatal sepsis occur in developing countries where the epidemiology of the bacteria causing neonatal infections are not clear, thus, the choice of empiric therapy needs to be individualized for different regions (15). It has been stated that empirical use of cefotaxime for neonatal sepsis may lead to increased bacterial resistance, and also increased risk of mortality $(4,19)$. Antibiotic stewardship has been proven as a successful strategy to curb irrational antibiotic usage for inpatients, stewardship needs to focus on regular surveillance of antibiotic usage, correct antibiotic choices (narrow spectrum vs. broad spectrum antibiotics), and prevention of prolonged continuation of antibiotics when cultures are negative in a well-looking infant (17). This survey reveals widespread use of broad-spectrum antibiotics and vancomycin in the neonatal units of Iranian hospitals and highlights the need for stringent antibiotic stewardship at these units.

\section{Acknowledgments}

This study was supported by the pediatric infectious disease research center, mofid children hospital, Shahid Beheshti University of Medical Sciences of Tehran, Iran.

\section{Footnote}

Financial Disclosure: The author(s) declared no potential conflicts of interest with respect to the research, authorship, and/or publication of this article.

\section{References}

1. Antimicrobial resistance: revisiting the "tragedy of the commons”. Bulletin World Health Organization. 2010;88(11):805-6. doi: 10.2471/blt.10.031110.

2. Carlet J, Pulcini C, Piddock LJ. Antibiotic resistance: a geopolitical issue. Clin Microbiol Infect. 2014;20(10):949-53. doi: 10.1111/14690691.12767. [PubMed: 25040923].

3. Goossens H.Antibiotic consumption and link to resistance. Clin Microbiol Infect. 2009;15 Suppl 3:12-5. doi: 10.1111/j.1469-0691.2009.02725.x. [PubMed: 19366364].

4. Tzialla C, Borghesi A, Serra G, Stronati M, Corsello G. Antimicrobial therapy in neonatal intensive care unit. Ital J Pediatr. 2015;41:27. doi: 10.1186/s13052-015-0117-7. [PubMed: 25887621].

5. Hosoglu S, Parlak Z, Geyik MF, Palanci Y. Critical evaluation of antimicrobial use-a Turkish university hospital example. J Infect Dev Ctries. 2013;7(11):873-9. doi: 10.3855/jidc.2921. [PubMed: 24240047].

6. Sharma M, Damlin A, Pathak A, Stalsby Lundborg C. Antibiotic Prescribing among Pediatric Inpatients with Potential Infections in Two Private Sector Hospitals in Central India. PLoS One. 2015;10(11):0142317. doi: 10.1371/journal.pone.0142317. [PubMed: 26540104].

7. Thu TA, Rahman M, Coffin S, Harun-Or-Rashid M, Sakamoto J, Hung NV. Antibiotic use in Vietnamese hospitals: a multicenter point-prevalence study. Am J Infect Control. 2012;40(9):840-4. doi: 10.1016/j.ajic.2011.10.020. [PubMed: 22341530]. 
8. Ang L, Laskar R, Gray JW. A point prevalence study of infection and antimicrobial use at a UK children's hospital. J Hosp Infect. 2008;68(4):372-4. doi: 10.1016/j.jhin.2008.01.030. [PubMed: 18353500].

9. Ciofi Degli Atti ML, Raponi M AE, Tozzi AE, Ciliento G, Ceradini J, Langiano T. Point prevalence study of antibiotic use in a paediatric hospital in Italy. Eurosurveillance. 2008;13(41):1-3.

10. Ansari F, Erntell M, Goossens H, Davey P. The European surveillance of antimicrobial consumption (ESAC) point-prevalence survey of antibacterial use in 20 European hospitals in 2006. Clin Infect Dis 2009;49(10):1496-504. doi:10.1086/644617. [PubMed: 19842976].

11. Zarb P, Amadeo B, Muller A, Drapier N, Vankerckhoven V, Davey P, et al. Identification of targets for quality improvement in antimicrobial prescribing: the web-based ESAC Point Prevalence Survey 2009. J Antimicrob Chemother. 2011;66(2):443-9. doi: 10.1093/jac/dkq430. [PubMed: 21084362].

12. Zarb P, Goossens H. European Surveillance of Antimicrobial Consumption (ESAC): value of a point-prevalence survey of antimicrobial use across Europe. Drugs. 2011;71(6):745-55. doi: 10.2165/11591180 000000000-00000. [PubMed: 21504250].

13. Versporten A, Sharland M, Bielicki J, Drapier N, Vankerckhoven V, Goossens $\mathrm{H}$, et al. The antibiotic resistance and prescribing in European Children project: a neonatal and pediatric antimicrobial webbased point prevalence survey in 73 hospitals worldwide. Pediatr Infect Dis J. 2013;32(6):242-53. doi: 10.1097/INF.0b013e318286c612. [PubMed 23838740].

14. Versporten A, Bielicki J, Drapier N, Sharland M, Goossens H, Arpec project group . The Worldwide Antibiotic Resistance and Prescribing in European Children (ARPEC) point prevalence survey: developing hospital-quality indicators of antibiotic prescribing for children.
J Antimicrob Chemother. 2016;71(4):1106-17. doi: 10.1093/jac/dkv418. [PubMed: 26747104].

15. Fahimzad A, Eydian Z, Karimi A, Shiva F, Sayyahfar S, Kahbazi M, et al. Surveillance of Antibiotic Consumption Point Prevalence Survey 2014: Antimicrobial Prescribing in Pediatrics Wards of 16 Iranian Hospitals. Arch Iran Med. 2016;19(3):204-9. [PubMed: 26923893].

16. Osowicki J, Gwee A, Noronha J, Britton PN, Isaacs D, Lai TB, et al. Australia-wide Point Prevalence Survey of Antimicrobial Prescribing in Neonatal Units: How Much and How Good? Pediatr Infect Dis J. 2015;34(8):185-90. doi: 10.1097/INF.0000000000000719. [PubMed: 25961896].

17. Cantey JB, Wozniak PS, Sanchez PJ. Prospective surveillance of antibiotic use in the neonatal intensive care unit: results from the SCOUT study. Pediatr Infect Dis J. 2015;34(3):267-72. doi: 10.1097/INF.0000000000000542. [PubMed: 25191849].

18. Seale AC, Obiero CW, Berkley JA. Rational development of guidelines for management of neonatal sepsis in developing countries. Curr Opin Infect Dis. 2015;28(3):225-30. doi: 10.1097/QCO.0000000000000163. [PubMed: 25887615].

19. Tripathi N, Cotten CM, Smith PB. Antibiotic use and misuse in the neonatal intensive care unit. Clin Perinatol. 2012;39(1):61-8. doi: 10.1016/j.clp.2011.12.003. [PubMed: 22341537].

20. Hariharan S, Chen D, Harry C, Ragobar R, Boodoosingh R, Gangoo C, et al. Antimicrobial prescription and usage in the neonatal intensive care units of a Caribbean country: a prospective observational study. J Neonatal Perinatal Med. 2013;6(4):325-31. doi: 10.3233/NPM-1372113. [PubMed: 24441089].

21. Sivanandan S, Soraisham AS, Swarnam K. Choice and duration of antimicrobial therapy for neonatal sepsis and meningitis. Int J Pediatr. 2011;2011:712150. doi: 10.1155/2011/712150. [PubMed: 22164179]. 